\title{
A Model for a Successful Implementation of Continuous Assessment in Limpopo Secondary Schools
}

\author{
Matome L Ramalepe \\ Limpopo Department of Education/University of South Africa \\ tetelo4life@ymail.com
}

\section{Doi:10.5901/mjss.2015.v6n1p578}

\begin{abstract}
Continuous Assessment (CASS) remains a seminal policy underlying South African curriculum despite notable curriculum changes that have transpired in this country since 2001. The widely acknowledged merit of CASS is that it assesses learners' strengths and weaknesses, this allows for a remedial support for slower learners. In practice, however, implementing CASS in schools is not without trouble due to several factors such as lack of monitoring and support, learner discipline and lack of parent involvement due to high illiteracy levels. There is currently no decisive action plan in South Africa to address these challenges. It is against this backdrop of lacking an explicit and immediate remedy to the problems inhibiting the practice of CASS in schools that the conceptual model is proposed. The conceptual model draws upon the recommendations of my study of the role of school managers in the implementation of CASS in Mopani district, Limpopo province. Therefore, drawing from literature and own study, the purpose of this article is to (1) explore the challenges hampering the implementation of CASS in schools and (2) uncover a model for unraveling the challenges that inhibit the implementation of CASS in schools.
\end{abstract}

Keywords: Continuous assessment, evaluation, monitoring, motivation, support

\section{Introduction}

The notion of continuous assessment was introduced in 2001 by the South African education department as the underlying policy of the National Curriculum Statement. This notion was brought in as an "ongoing diagnostic and schoolbased process that uses variety of assessment tools to measure learner performance" (Kapambwe \& Mulenga, 2008:2). Despite curriculum changes and developments that had transpired in South Arica, the notion of CASS continues to serve as a classroom strategy teachers apply to ascertain the knowledge, skills and values attained by learners using both informal and formal tasks, using different kinds of assessment methods and instruments. Undoubtedly, the move to introduce CASS or school-based assessment policy in South Africa was motivated by its widely documented benefits in the international literature. The widely recognised merits of CASS in teaching and learning include the following: (1) it develops knowledge, skills and values, (2) it promotes frequent classroom interactions, (3) it motivates and encourages learners, and (4) it allows constant assessment of learners' strengths and weaknesses and informs remedial programme (DoE, 2008a).

\section{Trends and Challenges of Implementing CASS in Schools}

Notwithstanding the benefits it offers to schools, the practice of CASS in schools is inhibited by major factors, mostly emanating from unique school contexts. The major problems working against the implementation of continuous assessment in South African schools are similar to those challenging the same policy in other parts of the world. These problems encompass large class sizes, staffing, remediation and enrichment, learner absenteeism, insufficient supply of teaching and learning resources, poor teacher networking, inadequate monitoring and feedback, unbearable administrative workload, lack of skills in test construction and record keeping by educators, lack of remedial instruction based on CASS, lack of moderation, and illiteracy levels of parents (Alausa, 2003:3, IAEA, 2006:4, Kapambwe \& Mulenga, 2008:6-7, Ramalepe, 2010, Quansah, 2005:2-4). These challenges hamper the implementation of CASS by adversely impacting three aspects of the implementation process, namely, designing, administration and evaluation. Designing aspect of implementation process of CASS involves development of lesson plans, assessment policies and test construction. It can be affected by factors such as lack of planning, teachers' negative attitude towards the CASS policy and their lack of skills required for the development of assessment tasks including tests. Factors negatively 
affecting administration aspect of the CASS implementation process include lack of moderation from the school management teams, lack of feedback from the SMT to teachers and from teachers to learners, learner discipline manifesting in learner absenteeism and other behavioural problems, lack of monitoring and support from the members of the SMT and lack of parent involvement and participation. Evaluation is the process informed by recorded data on the learning of learners; therefore, it can be extremely challenged by lack of meticulous record keeping. Combined, these challenges have a negative bearing on the implementation of CASS.

\section{Unveiling the Model for Addressing the Challenges Inhibiting Effective Implementation of CASS}

It is difficult and almost impossible to cultivate effective assessment under such inhibiting conditions. A growing concern is that there is currently a lack of explicit action plan to address these problems and a struggle to rethink a more effective perspective from which these problems may be dealt with. It is against this backdrop of an enormous challenge to find a working solution to the problems associated with the process of implementing CASS in schools that a model for school leaders is proposed. Successful leaders cannot simply accept negative factors but have to work hard to overcome them. Therefore, the proposed model serves to highlight a strategy school leaders can apply to enhance the implementation of CASS in their schools. The model is drawn upon the recommendations of my study of the role of school managers in the implementation of continuous assessment in Mopani District, Limpopo Province (Ramalepe, 2010). What emerges from a thorough scrutiny of these recommendations is that in order to reassert the value of continuous assessment in schools, the role of school managers in the implementation process should be detailed and clarified. An interesting conclusion drawn from these recommendations is that the role of school managers in the implementation of CASS should consists of five critical aspects. Therefore, the conceptual model (Figure 1) seeks to elucidate these significant ingredients and postulates their implications for the successful implementation of continuous assessment in schools. These aspects or ingredients are monitoring, support, motivation, resource provisioning and evaluation.

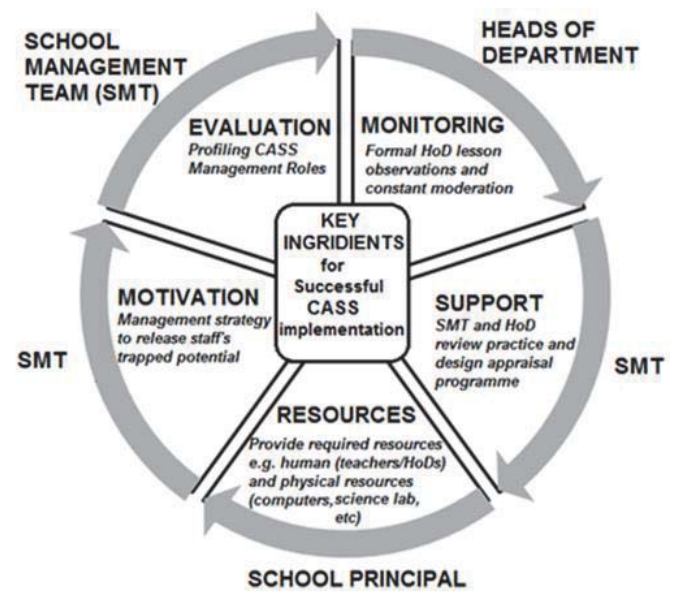

Figure 1 Conceptual model for successful implementation of CASS

\subsection{Monitoring}

The lack of monitoring emerged as a huge problem working against the cultivation of CASS in most schools. Thus, my study recommended that school managers should be relieved of administrative duties and other activities that increase their workload which distract them from monitoring and supporting teachers (Ramalepe, 2010). In line with the foregoing, the model proposes that it is commitment to monitoring of the teaching and learning processes that can ensure improvement in learner assessment. Monitoring is undertaken to establish whether teaching and learning are taking place in satisfactory way (Bush \& Glover, 2008). The heads of department or the SMTs need to monitor continuous or classroom assessment to ensure that assessment is not done once off, like in the form of a test or an examination, but takes place on a continuous bases. The model proposes two significant activities underlying the monitoring role of school managers which can improve the quality of assessment in the school, namely, moderation and lesson observations. 
Firstly, monitoring through moderation allows school managers to verify results of school-based assessment to ensure that assessment of the outcomes described in the National Curriculum Statement is fair, valid and reliable (LDoE, 2008).

According to Ramalepe (2010), moderation is the process of verifying that the requirements of continuous assessment are met by the teachers at school level. School-level moderation is an intensive internal process to ensure that teachers are supported throughout the process of implementing CASS. It usually consists of pre-administration (before the task is administered) and post-administration (after the task is administered. Pre-administration moderation serves the purpose of quality assurance in which the SMT uses a moderation tool to check the quality of assessment tasks (e.g. test or project) submitted by the teacher. In this moderation the SMT also check if assessment tasks are designed in accordance with the CASS guidelines and covers the prescribed content. In the post-administration moderation or term moderation, the SMT draws samples of ten percent of the total number of learners' portfolio of evidence and complete the moderation tool to check if assessment tasks were administered in accordance with the principles of good assessment. In this moderation, the school managers also check if the assessment evidence or learner scripts are marked according to the marking guidelines and marks are correctly totaled and recorded as per policy (IAEA, 2006).

Secondly, monitoring should involve visiting classrooms, observing teachers at work and providing them with feedback. O'Sullivan (2006) stresses that educational quality can only be improved if there is systematic observation of what is happening in the classroom. Observation is used to detect whether the lesson is progressing properly and reaching the learners and to assess whether and how the teacher assesses learner comprehension, for example through appropriate questioning techniques. The importance of lesson observations was stressed in O' Sullivan's (2006:253) report on the needs of Namibian teachers: "Lesson observations data were found to be particularly useful for needs assessment. They provided an insight into teachers' realities, their problems and training needs, which was not accessible using the other methods, for example, interviewing teachers about their needs". To effectively conduct lesson observations, the Heads of Department should be given a freedom to develop their own observation instruments which are linked to the specific needs of their subjects. Lesson observations should be accompanied by HoD's verbal and written feedback, in this way, they become both monitoring and development and it should take time every term.

\subsection{Support}

Support is usually an aspect that overlaps with monitoring and commitment to this aspect can enhance the implementation of continuous assessment in schools in two ways. Firstly, support implies that once areas of weakness are identified through monitoring, SMTs provide direct support to teachers to assist them to improve classroom instruction. Clearly, in more successful schools teachers will be provided with classroom support that includes providing them with coaches and mentors, and school leaders implemented whole group teacher discussions to help individual teachers solve problems. Furthermore, Coleman (2003:145) considers the importance of developing a supportive culture and states that "Promoting effective learning and teaching and encouraging a culture of learning have wide implications to those involved in management of schools". That is, school managers should focus on specific aspects of staff development by considering ways of improving the teaching styles of teachers. Mason (2004:21) states in managing instructional programmes, school managers should give guidance to educators on aspects such as modes of assessment, validity, reliability and objectivity of examinations, marking of papers, administration of internal and external examinations, and the relationship and influence of formative and summative assessment on teaching. Furthermore, the model suggests that school managers who desire success in implementing CASS should implement whole group teacher discussions to help individual to solve problems relating to CASS. In addition, school managers may provide novice teachers should provide novice teachers with classroom support which may include providing them with coaches and mentors.

\subsection{Resources}

The findings in my study highlighted that the issue of human, financial and teaching resources is indispensable for the implementation of continuous assessment (Ramalepe, 2010). With regard to human resource, four out of five school principals in my study indicated that in addition to their school management role they are forced to head department due to the shortage of HoDs in their schools. Amidst the crisis of the shortage of HoDs in schools, some school principals opted to use 'functional' heads of department to run departments though they were not recognised by the department of education. An important recommendation in this regard is that the department of education needs to speed up the process of filling up the promotional posts in schools where they exist. This process should take place at the beginning of 
the year or in the first term in order to avoid a situation where schools are left without managers in the second, third and fourth term in which learners' assessment become more demanding. An annual survey should be conducted to determine how school managers are in excess at their school staff establishments so that they may be quickly allocated to new schools. Another issue that emerged in this study with respect to resources was that some school principals were reluctant to provide teachers with required teaching resources. The lack of teaching resources such as chemicals to perform experiments or practical has a negative impact on the implementation of CASS. The model proposes that in order to successfully implement CASS, schools need school principals who are conscious about their roles as resource providers. Making provision for human, financial and physical resource will enable a creation of an atmosphere hospitable to the cultivation of continuous assessment in schools.

\subsection{Motivation}

The need for motivation emerged in my findings as a factor in schools that boasts staff morale and energises learners to achieve at higher levels. This resonate with the claim of Leithwood, Day, Sammons, Harris and Hopkins (2006) that school leaders improve teaching and learning indirectly and most powerfully through their influence on staff motivation, commitment and working conditions. In the same vein, Mason (2004) argues that motivation a management strategy to persuade people to change, to release staff's trapped potential and to bring out the best in people, to improve teaching and learning and to satisfy some psychological need in an individual for the benefit of both the individual and the school. It is believed that motivation has always been a missing link in the implementation process of the CASS policy in South Africa. Many unpleasant problems hampering the implementation of CASS developed as a result of the low motivational levels of teachers in schools. The low motivational levels might have been as a result of interplay of factors such as unbearable workloads, class size, lack of parent involvement and learners' behavioural problems. It is therefore, the SMT's responsibility to keep teachers moving energetically towards a goal or to work hard, even if the implementation task is difficult. When the school managers engage wittingly with staff motivation, the staff will become more productive, hence, securing triumph in the face of overwhelming challenges confronting the implementation of CASS. Motivation as an ingredient has a positive effect on the implementation of CASS because motivated employees are always looking for better ways of doing their job and will usually find them and motivated workers are usually concerned about quality (Anderson \& Kyprianou, cited in Steyn \& Van Niekerk 2007).

\subsection{Evaluation}

Evaluation is another important tool to enhance the quality of teaching and learning (Bush, Joubert, Kiggundu \& Van Rooyen, 2008). While monitoring seeks to assess the ways in which the teaching plans are put into effect, evaluation seek to assess the impact of teaching- learning processes (e.g. assessment) at a more strategic level. In the context of my article, evaluation plays two significant roles in order to inform school management teams about the effectiveness or lack of it in their schools. Firstly, evaluation seeks to compare within-the-school performance between the five "traditional leadership and management roles" (Ramalepe, 2010:39), that is, to assess which roles are effectively performed by school managers in comparison to other roles in the process of implementing continuous assessment. To achieve this, I recommended that a system should be developed which I referred to it as " 5 roles - School Profiling System." This system involves determining "minimum factors" - those roles which are least performed among the five roles postulated in my study as roles that have influence in the cultivation of CASS in schools, namely, instructional leadership, parent management, learner management, staff motivation and monitoring and support (Ramalepe, 2010). The "minimum factors" adversely hinder the implementation of CASS in the classroom. However, the term "minimum factor" does not necessarily suggest that the school managers are bad in certain roles; it simply means these are "low point" roles on which school managers are not showing full commitment to and the process of implementing CASS is hampered as a result.

For example, Bush and Heystek's (2006) study found that school managers do not conceptualise their role as instructional leaders who manage teaching and learning. Instead, their time is largely consumed by administrative activities (Chisholm, Hoadley \& Kivilu, 2005). In this case, "instructional leadership" is a "minimum factor" - a role that is least performed and serves as an inhibitor in the process of implementing CASS. To improve the implementation of CASS and eliminating factors inhibiting its implementation, school managers need to transform their attitude towards these "low point" roles. By committing fully to these roles will bring lasting progress in the teaching and learning processes in the school. An example of how these roles can be assessed is provided in figure 2 below: 


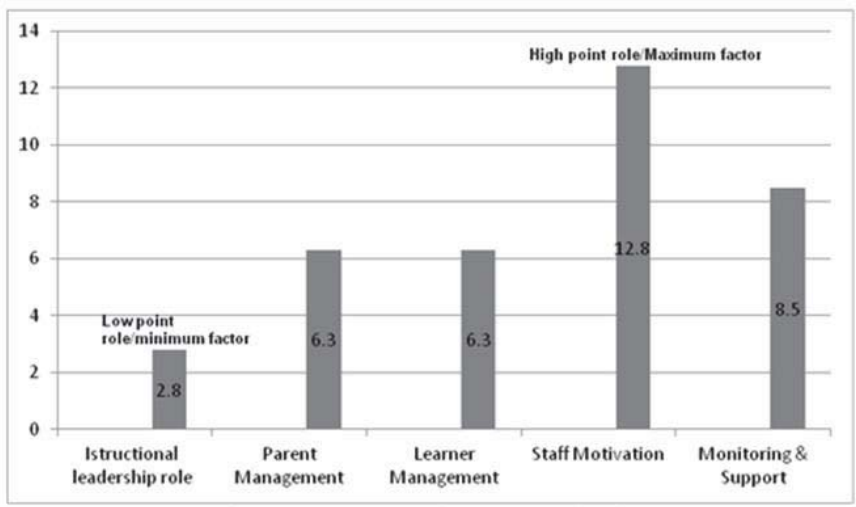

Figure 2 School Profiling System for traditional roles of school managers

In my example above, instructional leadership is a "low point" role which has a minimum impact on the implementation process of CASS and this role needs an urgent attention. The "high point" role is staff motivation, which means that it has a maximum influence on the cultivation of continuous assessment. Whenever a "low point" role is identified, intervention in the form of training or any other means should be implemented to enhance performance in that role. Secondly, evaluation seeks to compare the performance between subjects within the same school to determine the effect of continuous assessment on learner outcomes. Comparing results within a single school is straightforward and much meaningful because "If the same group of learners performs well in one subject and badly in another, this must be due to internal factors, not the external context" (DoE, 2008b:66). This is what Reynolds (2007) term 'within-school variation' and manifests several reasons which may include differences in quality or commitment of educators to continuous assessment, differences in commitment of HoDs in their monitoring and support role, and attitudes of learners and parents.

Moreover, the variation between the subjects within the same school provide an opportunity for teachers to interact and learn strategies applied by those teachers and managers who are able to succeed in their subjects despite contextual barriers to teaching and learning. This evaluation also reflects different subject cultures and can allow different departments to swap practice. For example, the HoD for the poor performing department may request from a department with a high-quality observational system an observation tool to use in his or her department. This approach will reduce within school variation. Accordingly, Reynolds (2007:18) argues that the reduction of within-school variation requires: attempting to specify the core classroom-related teacher behaviours, encouraging discussion of teaching in departments and across the school, attempting greater consistency in teaching behaviours and especially in the expectations of learners, within and across departments.

\section{Conclusion}

The proposed model advocates that due to the challenges confronting continuous assessment, the transformation of schooling contexts has become urgent in our schools. It proposes that the essential ingredients for successful implementation of continuous assessment and for addressing these challenges are monitoring, support, resources, evaluation and motivation. School managers should develop a programme to monitor educators' practices in a systematic way and provide constructive feedback as a means by which they support the teachers. Support may take several forms such as motivation of the teaching staff and developing appraisal programme to address areas of their weaknesses. They should also conduct within school variation (evaluate school outcomes) to determine reasons why other subjects are performing well while others are performing badly. Furthermore, they need to determine which management roles are low point roles, roles which are least performed by school managers. It is imperative, therefore, to note in this article that the implementation of this model requires commitment from both the school managers and teachers in order to realised the expected outcomes. 


\section{References}

Alausa, Y.A. 2003. CASS in our schools: Advantages and problems. Retrieved from http://www.nied.educational/publication/journal/ journal 9 (accessed 21 July 2009).

Bush, T., Joubert, R., Kiggundu, E. \& Van Rooyen, J. 2008. Leading and Managing Literacy and Numeracy, Johannesburg, The Zenex Foundation.

Bush, T., \& Heystek, J. 2006. School leadership and management in South Africa: Principals' perceptions. International Studies in Educational Administration, 34(3), 63-76.

Chisholm, L., Hoadley, U., \& Kivilu, M. 2005. Educator Workload in South Africa, Report Prepared for Educator Labour Relations Council, Pretori, Human Sciences Research Council.

Coleman, M. 2003. School Effectiveness. In Thurlow, M.M., Bush, T. and Coleman, M. (Eds.), Leadership and Strategic Management in South African Schools, London: Commonwealth Secretariat.

Department of Education. 2008a. Subject Assessment Guidelines, Physical Sciences, grades 10 - 12 (General). Pretoria. Government Printer.

Department of Education. 2008b. Managing teaching and learning. Core model, ACE SML. Pretoria, South Africa.

IAEA. 2006. Can Statistical and Qualitative Modes of Moderation Co-exist in a Model for the Quality Assurance of School-based Assessment - A South African Perspective. Paper submitted at the 2006 IAEA Annual Conference. Retrieved from http://www.iaea.org.za (accessed 21 July 2009).

Kapambwe, W.M. \& Mulenga, M.G. 2008. The Implementation of School Based Assessment (CA) in Zambia. The 2008 IAEA Conference. Retrieved from http://www.iaea.org.za. (accessed 21 July 2009).

Leithwood, K., Day, C., Sammons, P., Harris, A. \& Hopkins, D. 2006. Seven strong claims about successful school leadership. London: Department for Education and Skills.

Limpopo Department of Education (LDoE). 2008. Guidelines for the implementation of Continuous Assessment (CASS) in the Further Education and Training (FET) Band [Grade 10, 11, 12]. Government Printers.

Mason, T. 2004. Curriculum 2005. Revised National Curriculum Statement: Intermediate phase, School Management Teams. Johannesburg: Wits.

O' Sullivan, M. 2006. Lesson observation and quality in primary education as contextual teaching and learning processes. International Journal of Educational Development, 26, 246-260.

Quansah, K.B. 2005. Continuous Assessment Handbook. Ghana education service publication. Accra: BECAS project publication.

Ramalepe, M.L. 2010. The role of school managers in the implementation of continuous assessment in the Further Education and Training Band in Mopani District, Limpopo Province. Unpublished Med. dissertation, University of South Africa.

Reynolds, D. 2007. Schools Learning from their Best: The Within School Variation Project, Nottingham, NCSL.

Steyn, G.M. \& Van Niekerk, E.J. 2007. Human Resource Management in Education. University of South Africa, Pretoria. 